\title{
ON STABLE NOETHERIAN RINGS
}

\author{
BY
}

\author{
ZOLTAN PAPP
}

ABSTRACT. A ring $R$ is called stable if every localizing subcategory of $R^{M}$ is closed under taking injective envelopes. In this paper the stable noetherian rings are characterized in terms of the idempotent kernel functors of $R^{M}$ (O. Goldman [5]). The stable noetherian rings, the classical rings (Riley [11]) and the noetherian rings "with sufficiently many two-sided ideals" (Gabriel [4]) are compared and their relationships are studied. The close similarity between the commutative noetherian rings and the stable noetherian rings is also pointed out in the results.

1. Introduction. In [4] Gabriel calls a localizing subcategory stable if it is closed under injective envelopes, and proves that a commutative noetherian ring $R$ is stable, that is, every localizing subcategory of ${ }_{R} M$ is stable. A commutative noetherian ring $R$ has another interesting property, namely, the prime ideals of $R$ and the isomorphism classes of the indecomposable injective modules are in oneto-one correspondence. The class of (not necessarily commutative) noetherian rings having this property has been studied by Michler [10], Lambek and Michler [8], Krause [7] and also by Gabriel [4] whose terminology was "rings with sufficiently many two-sided ideals", SMI-rings for a short notation. Gabriel has also proved in [4] that a stable ring $R$ satisfies the Artin-Rees property for left ideals. The rings with the Artin-Rees property were named classical rings by Riley [11] and he has proved that a noetherian ring $R$ is classical if and only if the tertiary decomposition theory of Lesieur and Croisot [9] coincides with the primary decomposition theory. In this paper we characterize the stable neotherian rings and begin to study the properties and the connections of the following classes of noetherian rings: stable rings, SMI-rings and classical rings.

All the rings $R$ considered here are supposed to have identity element, and each $R$-module is considered as a unitary left $R$-module. $E(M)$ denotes the injective envelope of an $R$-module $M$, and ${ }_{R} M$ is used to denote the category of left $R$ modules. For terminology and results we refer to Sharpe and Vamos [12], Anderson and Fuller [1] and also to Goldman [5], whose idempotent kernel 1974.

Presented to the Society, January 23, 1975; received by the editors November 18,

AMS (MOS) subject classifications (1970). Primary 16A46, 16A48, 18E40; Secondary 16A64, 16 A66.

Key words and phrases. Stable localizing subcategory, kernel functor, prime kernel functor, stable ring, SMI-ring, classical ring. 
functors will be one of our main tools. In the following we review the relevant definitions of Goldman's paper [5].

A functor $\tau:{ }_{R} M \rightarrow{ }_{R} M$ is called an idempotent kernel functor if the following are satisfied.

(1) For every $M \in_{R} M, \tau(M)$ is a submodule of $M$.

(2) If $f \in \operatorname{Hom}_{R}(M, N)$, then $f(\tau(M)) \subseteq \tau(N)$ and $\tau(f)$ is the restriction of $f$ to $\tau(M)$.

(3) If $M^{\prime} \subseteq M$, then $\tau\left(M^{\prime}\right)=M^{\prime} \cap \tau(M)$.

(4) $\tau(M / \tau(M))=0$.

In our terminology "kernel functor" will always mean "idempotent kernel functor". To each $R$-module $K$ a kernel functor $\tau_{K}$ can be assigned by the following definition:

$$
\tau_{K}(M)=\bigcap\left\{\operatorname{Ker} \varphi ; \varphi \in \operatorname{Hom}_{R}(M, E(K))\right\} \quad \text { for all } M \in{ }_{R} M .
$$

$\tau_{K}$ is called the associated kernel functor to $K$. Given a kernel functor $\tau$, we say that $M$ is $\tau$-torsion-free ( $\tau$-torsion) module if $\tau(M)=0(\tau(M)=M)$. The $\tau$-torsionfree module $W \neq 0$ is a supporting module for $\tau$ if $\tau\left(W / W^{\prime}\right)=W / W^{\prime}$ for each $0 \neq W^{\prime} \subseteq W$. The kernel functor $\pi$ of ${ }_{R} M$ is called prime if $\pi=\tau_{W}$ for some $\pi$ supporting module $W$. It has been shown by Michler [10, Corollary 1.2] that a kernel functor $\pi$ is prime if and only if $\pi=\tau_{F}$ for an indecomposable injective $R$-module $F$, which is (up to isomorphism) uniquely determined by $\pi$.

Given a kernel functor $\tau$, the $\tau$-torsion modules form a localizing subcategory $T$ of ${ }_{R} M$ in the sense of Gabriel [4].

There is a partial order relation in the set of kernel functors of ${ }_{R} M$; if $\sigma(M) \subseteq \tau(M)$ for all $M \in_{R} M$, then we say $\sigma \leqslant \tau$. It is easy to see that $\sigma \leqslant \tau$ if and only if for every $M \in_{R} M, \sigma(M)=M$ implies $\tau(M)=M$.

2. Stable noetherian rings. If $R$ is a commutative noetherian ring, $P$ and $Q$ are prime ideals of $R$, then by Proposition 4.21 of [12]

$$
\operatorname{Hom}_{R}(E(R / Q), E(R / P)) \neq 0
$$

if and only if $Q \subseteq P$, which, in turn, is true if and only if $\tau_{E(R / P)} \leqslant \tau_{E(R / Q)}$. In this section we generalize this result for certain noncommutative noetherian rings $R$. Namely, we show that the above proposition characterizes the stable noetherian rings among the arbitrary noetherian rings and, as a result, there exists a partial order on the set of isomorphism classes of indecomposable injective $R$ modules paralleling the partial order relation of prime ideals of the commutative noetherian ring given by the inclusion relation. In the case when the noetherian ring $R$ is an SMI-ring the criterion preserves the exact form of Proposition 4.21 of [12] and the stability of the commutative noetherian rings can be obtained as a consequence. 
DEFinition 1. A kernel functor $\tau$ is stable if the localizing subcategory $T=\left\{M ; M \in_{R} M, \tau(M)=M\right\}$ is closed under injective envelopes.

Definition 2. A ring $R$ is stable if every kernel functor $\tau$ of ${ }_{R} M$ is stable.

Our first result shows that the stability of the ring $R$ depends on the stability of the prime kernel functors of ${ }_{R} M$.

THEOREM 1. A noetherian ring $R$ is stable if and only if every prime kernel functor $\pi$ of $R_{R} M$ is stable.

Proof. Only the "if" part needs explanation. Assume that every prime kernel functor is stable. Given a kernel functor $\sigma$, then $\sigma=\tau_{F}$ for some injective module $F$ (Goldman [5, Theorem 5.3]). If $F=\bigoplus_{i \in I} F_{i}$, where $F_{i}(i \in I)$ are indecomposable injective $R$-modules, then $\tau_{F_{i}}(i \in I)$ are prime kernel functors and $\sigma=\tau_{F}=\bigcap_{i \in I} \tau_{F_{i}}$ in the sense that, for every $M \in{ }_{R} M, \sigma(M)=\bigcap_{i \in I} \tau_{F_{i}}(M)$. Therefore, the stability of the prime kernel functors $\tau_{F_{i}}(i \in I)$ implies that $\sigma$ is stable.

Given an arbitrary noetherian ring $R$ and the indecomposable injective modules $F$ and $G$, then $\tau_{G} \leqslant \tau_{F}$ always implies that $\operatorname{Hom}_{R}(F, G) \neq 0$. The relations $0 \subseteq \tau_{G}(F) \subseteq \tau_{F}(F)=0$ show that

$$
0=\tau_{G}(F)=\bigcap\left\{\operatorname{Ker} \varphi ; \varphi \in \operatorname{Hom}_{R}(F, G)\right\},
$$

hence we must have $\operatorname{Hom}_{R}(F, G) \neq 0$. On the other hand, $\operatorname{Hom}_{R}(F, G) \neq 0$ does not necessarily imply $\tau_{G} \leqslant \tau_{F}$. We will show it in an example after Theorem 9. Moreover, the noetherian rings for which the assertions $\tau_{G} \leqslant \tau_{F}$ and $\operatorname{Hom}_{R}(F, G) \neq 0$ are equivalent for any two indecomposable injectives $F$ and $G$ are exactly the stable noetherian rings. This is included in the following theorem.

THEOREM 2. Let $R$ be a noetherian ring, then the following are equivalent.

(a) $R$ is a stable ring.

(b) Given any kernel functor $\tau$ of ${ }_{R} M$, then every indecomposable injective $R$-module $F$ is either $\tau$-torsion or $\tau$-torsion free.

(c) For any two indecomposable injective $R$-modules $F$ and $G, \operatorname{Hom}_{R}(F, G)$ $\neq 0$ if and only if $\tau_{G} \leqslant \tau_{F}$.

Proof. (a) $\Rightarrow(b)$ is obvious.

(b) $\Rightarrow$ (c). Given the indecomposable injective $R$-modules $F$ and $G$, it is enough to show that $\operatorname{Hom}_{R}(F, G) \neq 0$ implies $\tau_{G} \leqslant \tau_{F}$. If $\operatorname{Hom}_{R}(F, G) \neq 0$, then $F$ is not $\tau_{G}$-torsion, hence $\tau_{G}(F)=0$ by assumption (b). Let $M \in_{R} M$ with $\tau_{G}(M)=M$, and let $\varphi \in \operatorname{Hom}_{R}(M, F)$, then the relations $\varphi(M)=\varphi\left(\tau_{G}(M) \subseteq\right.$ $\tau_{G}(F)=0$ show that $\varphi=0$ which implies that $\tau_{F}(M)=0$.

(c) $\Rightarrow$ (a). By Theorem 1, it is enough to prove that every prime kernel functor is stable. Let $\pi$ be a prime kernel functor, then $\pi=\tau_{G}$ for some 
indecomposable injective $R$-module $G$. Given the $\pi$-torsion module $M$, we have the decomposition $E(M)=\bigoplus_{i \in I} F_{i}$, where $F_{i}(i \in I)$ are indecomposable injective $R$-modules. $E(M)$ is $\pi$-torsion if and only if all the $F_{i}(i \in I)$ are $\pi$-torsion. Since the uniform modules $M_{i}=F_{i} \cap M \neq 0$ ( $\left.i \in I\right)$ are $\pi$-torsion modules, our task is reduced to showing that if a uniform module $U \neq 0$ is $\pi$-torsion, then $F=E(U)$ is also $\pi$-torsion. Assume otherwise, then $\operatorname{Hom}_{R}(F, G) \neq 0$ and consequently $\tau_{G} \leqslant \tau_{F}$ which leads to the contradiction $0 \neq U \subseteq \pi(F)=\tau_{G}(F) \subseteq \tau_{F}(F)=0$.

REMARK. Given a simple $R$-module $V$ and a kernel functor $\tau$, then $\tau(V)=$ 0 or $\tau(V)=V$. Condition (b) of the theorem states that the same property for indecomposable injective modules characterizes the stable noetherian rings.

Let $R$ be a stable noetherian ring. Given the indecomposable injective $R$ modules $F$ and $G$ we define the following relation: $G \leqslant F$ if $\operatorname{Hom}_{R}(F, G) \neq 0$. This relation is reflexive, and Theorem 2 implies that it is also transitive. If $G \leqslant F$ and $F \leqslant G$, then, by Theorem $2, \tau_{F}=\tau_{G}$, thus Proposition 1.1 of Michler [10] shows that $G \cong F$, hence the relation is antisymmetric. Therefore we have the following result.

THEOREM 3. Let $R$ be a stable noetherian ring. The relation given above is a partial order on the set of isomorphism classes of indecomposable injective $R$-modules.

Let the rings $R$ and $S$ be (Morita) equivalent, that is, there exist functors F: ${ }_{R} M \rightarrow{ }_{S} M$ and $G:{ }_{S} M \rightarrow{ }_{R} M$ such that $\mathbf{G F} \cong \mathbf{1}_{R}$ and $\mathbf{F G} \cong \mathbf{1}_{S}$ (natural isomorphisms) where $1_{R}$ and $1_{S}$ are the identity functors on ${ }_{R} M$ and ${ }_{S} M$ respectively. If $H$ is an injective $R$-module, then $\mathrm{F}(H)$ is an injective $S$-module and given any $M \in_{R} M$,

$$
\operatorname{Hom}_{R}(M, H) \cong \operatorname{Hom}_{S}(\mathrm{~F}(M), \mathbf{F}(H))
$$

(Proposition 21.2 of [1]). This shows that $M$ is $\tau_{H}$-torsion if and only if $\mathbf{F}(M)$ is $\tau_{\mathrm{F}(H)}$-torsion. Combining this result with Proposition 21.6-(6) of [1] the following result readily follows.

THEOREM 4. The stability of a ring is a Morita-invariant property, i.e. it is preserved by the equivalence of rings.

This small excursion into category theory can be extended, since the stability of a ring $R$ is defined via the category of the left $R$-modules ${ }_{R} M$. One can prove the analog form of Theorem 2 for every abelian category which is locally noetherian and has sufficiently many injectives. (For terminology see Gabriel [4].) The stability of a category is defined in the same way as in the special case of a module category. Consider the categories $C_{1}$ and $C_{2}$, endowed with all the properties we need to define stability (e.g. consider module categories), 
and form the product category $C_{1} \times C_{2}$. Then we have that $E\left(U_{1} \times U_{2}\right) \cong$ $E\left(U_{1}\right) \times E\left(U_{2}\right)$ and

$$
\operatorname{Hom}_{C_{1} \times c_{2}}\left[\left(U_{1}, U_{2}\right),\left(V_{1}, V_{2}\right)\right] \cong \operatorname{Hom}_{C_{1}}\left(U_{1}, V_{1}\right) \times \operatorname{Hom}_{C_{2}}\left(U_{2}, V_{2}\right)
$$

for $U_{1}, V_{1} \in C_{1}$ and $U_{2}, V_{2} \in C_{2}$. It follows that any kernel functor $\sigma$ of $C_{1} \times C_{2}$ has the form $\sigma=\tau_{H_{1} \times H_{2}}$ for some injective objects $H_{1} \in C_{1}$ and $H_{2}$

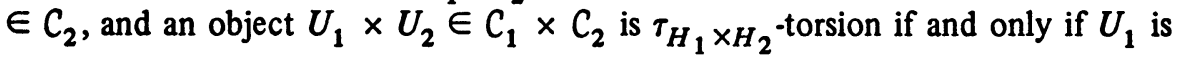
$\tau_{H_{1}}$-torsion and $U_{2}$ is $\tau_{H_{2}}$-torsion. Thus we can deduce the following result.

THEOREM 5. The product category $C_{1} \times C_{2}$ is stable if and only if $C_{1}$ and $\mathrm{C}_{2}$ are stable categories.

By [1, Exercise 21.5] we have that the product category $R_{1} M \times{ }_{R_{2}} M$ is equivalent to the category $R_{1} \oplus R_{2} M$, where $R_{1} \oplus R_{2}$ is the (ring) direct sum of the ring $R_{1}$ and $R_{2}$. This leads to the following

THEOREM 6. If $R=R_{1} \oplus R_{2} \oplus \cdots \oplus R_{n}$, finite (ring) direct sum of the rings $R_{1}, R_{2}, \ldots, R_{n}$, then $R$ is stable if and only if $R_{1}, R_{2}, \ldots, R_{n}$ are stable rings.

These results enable us to construct a number of examples of noncommutative, noetherian stable rings. Since the commutative noetherian rings are stable, Theorems 4 and 6 imply that any finite direct sum of the full matrix rings over commutative, noetherian rings is stable.

Consider a noetherian ring $R$ which has the property that the isomorphism classes of the indecomposable injective $R$-modules and the prime ideals of $R$ are in one-to-one correspondence, i.e. $R$ is a noetherian SMI-ring. In this particular case Theorem 2 has the same form as Proposition 4.21 of [12].

THEOREM 7. Let $R$ be a noetherian SMI-ring. Then $R$ is stable if and only if, for any two prime ideals $P$ and $Q, Q \subseteq P$ is equivalent to $\operatorname{Hom}_{R}(E(R / Q), E(R / P))$ $\neq 0$.

Proof. Let $R$ be a stable ring, and let $P$ and $Q$ be prime ideals of $R$. The relation $Q \subseteq P$ clearly implies that $\operatorname{Hom}_{R}(E(R / Q), E(R / Q)) \neq 0$. On the other hand, if $\operatorname{Hom}_{R}(E(R / Q), E(R / P)) \neq 0$, then $\operatorname{Hom}_{R}(F, G) \neq 0$, where $E(R / P)=$ $\bigoplus_{i=1}^{m} G_{i}, E(R / Q)=\bigoplus_{j=1}^{n} F_{j}, G_{i} \cong G(i=1, \ldots, m), F_{j} \cong F(j=1, \ldots, n)$, and $F$ and $G$ are indecomposable injective $R$-modules, because $E(R / P)$ and $E(R / Q)$ are isotopic injective modules (Gabriel [4, p. 421]). The stability of $R$ implies that $\tau_{E(R / P)}=\tau_{G} \leqslant \tau_{F}=\tau_{E(R / Q)}$, therefore, by Proposition 3.3, Definition 3.4 and Lemma 3.5 of Beachy [2], we have $Q \subseteq P$ as required.

Conversely, assume the conditions of the theorem and let $F$ and $G$ be indecomposable injective $R$-modules with $\operatorname{Hom}_{R}(F, G) \neq 0$. Since $R$ is a noetherian 
SMI-ring, there are uniquely associated prime ideals $P$ and $Q$ to $G$ and $F$ respectively such that the following are satisfied: $E(R / P)=\bigoplus_{i=1}^{m} G_{i}$ and $E(R / Q)=\bigoplus_{j=1}^{n} F_{j}$ where $G_{i} \cong G(i=1, \ldots, m)$ and $F_{j} \cong F(j=1, \ldots, n)$. (See Theorem 3.9 and Corollary 3.11 of [8].) It follows that $\operatorname{Hom}_{R}(E(R / Q), E(R / P)) \neq 0$, hence $Q \subseteq P$ by the assumption of the theorem. Now we apply again Lemma 3.5, Definition 3.4 and Proposition 3.3 of [2], in that order, and the conclusion is that $\tau_{E(R / P)} \leqslant \tau_{E(R / Q)}$, hence $\tau_{G} \leqslant \tau_{F}$ which proves, with the help of Theorem 2 , that $R$ is stable.

When $R$ is a commutative noetherian ring, then the conditions of Theorem 7 are satisfied, so we have Gabriel's result. (Gabriel [4, p. 428].)

COROLlaRY. If $R$ is a commutative, noetherian ring, then $R$ is stable.

3. Classical rings. In [11] Riley has named a ring $R$ a classical ring if each submodule of a finitely generated $R$-module $M$ may be written as a finite intersection of primary submodules of $M$. (For terminology we refer to Herstein [6] and Riley [11].) A noetherian ring $R$ is classical if and only if it has the ArtinRees property, that is, given a left ideal $L$, then for every positive integer $n$ and ideal $A$ of $R$, there exists a positive integer $m$ such that $A^{m} \cap L \subseteq A^{n} L$. It was proved by Gabriel [4] that the stable rings are classical. The following result is the converse of this theorem for noetherian SMI-rings.

THEOREM 8. A noetherian SMI-ring $R$ is classical if and only if it is stable.

Proof. The "if" part was proved by Gabriel [4]. Assume that the noetherian SMI-ring $R$ is classical and $F$ and $G$ are indecomposable injective $R$ modules with uniquely associated prime ideals $Q$ and $P$ respectively. Let $\operatorname{Hom}_{R}(F, G) \neq 0$ and $0 \neq \varphi \in \operatorname{Hom}_{R}(F, G)$. If $K=\operatorname{Ker} \varphi$, then $Q=t-\operatorname{rad}(0)$ $\subseteq t-\operatorname{rad}(K)=Q_{1}$ because $R$ is classical. $(t-\operatorname{rad}(N)$ is the tertiary radical of $N$ in an $R$-module $M$, see Herstein [6, p. 112] for results and terminology.) Since $K$ is an irreducible and consequently tertiary submodule of $F$, it is also primary because $R$ is classical. Then Lemma 6.9 of Herstein [6] implies that there exists an integer $n$ such that $Q_{1}^{n} F \subseteq K$. This shows that $Q^{n} \varphi(F) \subseteq \varphi\left(Q^{n} F\right) \subseteq \varphi\left(Q_{1}^{n} F\right)$ $\subseteq \varphi(K)=0$, therefore, $Q^{n} \subseteq P$, hence $Q \subseteq P$. By Theorem 7 we have that $R$ is stable.

As an application, we prove the following result which contains the properties of the artinian stable rings.

THEOREM 9. Let $R$ be an artinian ring, then the following are equivalent.

(a) $R$ is a stable ring.

(b) $R$ is a classical ring.

(c) $R$ is an " $H$-ring", that is, for the simple $R$-modules $U$ and $V$, $\operatorname{Hom}_{R}(E(U), E(V)) \neq 0$ if and only if $U \cong V$. (See Sharpe and Vamos $[12$, p. 110].) 
(d) $R$ is the direct sum of finitely many primary artinian rings. (Primary ring is one which modulo its Jacobson radical is a simple artinian ring.)

(e) For the nonisomorphic simple $R$-modules $U$ and $V, \operatorname{Ext}(V, U)=0$.

Proof. (a) $\Rightarrow$ (b). Gabriel $[4$, p. 427].

(b) $\Rightarrow$ (d). Riley [11, Proposition 7.5].

(d) $\Rightarrow$ (a). A primary artinian ring $S$ has only one prime ideal. It is also an SMI-ring [4, p. 423], hence all indecomposable injective modules are isomorphic to each other. Therefore, $S$ is stable by Theorem 2, and Theorem 6 implies that any finite direct sum is also stable.

(a) $\Rightarrow$ (e). Dickson $[3$, p. 13].

(e) $\Rightarrow$ (c). Since $R$ is an artinian ring, if $F$ is an indecomposable injective $R$-module, then $F=E(U)$, where $U$ is simple. Let $F^{*}$ be an arbitrary finitely generated submodule of $F$, then $U \subseteq F^{*}$ and we have a composition series $U=$ $F_{0} \subset F_{1} \subset \cdots \subset F_{n}=F^{*}$, where $F_{i} / F_{i-1}(i=1, \ldots, n)$ are simple $R$-modules. Since every submodule of $F$ is indecomposable, and, by assumption, $\operatorname{Ext}(V, U)=$ 0 for every simple $V$ with $V \neq U$, we have that $F_{i} / F_{i-1} \cong U(i=1, \ldots, n)$. This implies that $F^{*}$ is $\tau_{E(V)}$-torsion, hence $F$ is $\tau_{E(V)}$-torsion whenever $V \neq U$. Therefore, $\operatorname{Hom}_{R}(E(U), E(V)) \neq 0$ if and only if $V \cong U$.

(c) $\Rightarrow$ (a). Let $F$ and $G$ be indecomposable injective modules, then $F=$ $E(U)$ and $G=E(V)$ with $U$ and $V$ are simple. If $\operatorname{Hom}_{R}(F, G) \neq 0$, then by (c) $U \cong V$, hence the equalities $\tau_{F}=\tau_{U}=\tau_{V}=\tau_{G}$ imply that $R$ is stable via Theorem 2.

EXAMPLES. A noetherian, simple integral domain which is not a skewfield is an example for a classical but not SMI-ring. If $Q$ is the field of rational numbers and $R$ is the algebra over $Q$ defined by the elements $e_{1}, e_{2}$ and $n$ with the relations $e_{1}^{2}=e_{1}, e_{2}^{2}=e_{2}, n^{2}=0, e_{1} e_{2}=e_{2} e_{1}=0, e_{1} n=n e_{2}=n$ and $n e_{1}=$ $e_{2} n=0$, then $R$ is an artinian ring, hence an SMI-ring. It was shown by Riley $[11$, p. 188] that $R$ is not a classical ring, hence it is not a stable ring either. It follows from Theorem 8 that if a ring $R$ is classical and an SMI-ring, then $R$ is stable. One can ask whether or not the converse is true, namely, whether the stability of a noetherian ring $R$ implies that $R$ is an SMI-ring.

REMARK. It can be seen from the results of this paper that the stable noetherian rings share a number of properties with the commutative, noetherian rings. Finally, we point out one more common feature. In the Lasker-Noether primary decomposition of the submodule $N$ of the finitely generated module $M$ the components belonging to prime ideals which are minimal in the set of associated prime ideals of $N$ in $M$ are independent of the primary decomposition. Given the noetherian ring $R$ and a finitely generated $R$-module $M$, consider the Gabriel-Goldman generalization of the primary decomposition theory ([4] and 
[5]). Let $\Omega=\left\{F_{1}, \ldots, F_{n}\right\}$ be the set of associated injectives $\left(\left\{\tau_{F_{1}}, \ldots\right.\right.$, $\left.\tau_{F_{n}}\right\}$ is the associated prime kernel functors) of the submodule $N$ in $M$, then $G \in \Omega$ is called isolated if $\operatorname{Hom}_{R}(F, G)=0$ for $F \in \Omega, F \neq G$. It is proved in $[12$, p. 96$]$ that the $G$-isotopic component ( $\tau_{G}$-primary component) is independent of the decomposition. However, it cannot be assured that there exist isolated associated injective modules in the general case. For stable noetherian rings Theorem 2 of this paper provides the existence of isolated associated injectives, since every associated indecomposable injective $G$ of $N$ in $M$ is isolated if the prime kernel functor $\tau_{G}$ is maximal in the set of associated kernel functors of $N$ in $M$. (For more detail see Sharpe and Vamos [12].) As a particular case, when $R$ is an artinian ring the primary decomposition of a submodule $N$ of a finitely generated $R$-module $M$ is unique.

\section{REFERENCES}

1. F. W. Anderson and Kent R. Fuller, Rings and categories of modules, Graduate Texts in Math., no. 13, Springer-Verlag, New York, 1974.

2. J. A. Beachy, On maximal torsion radicals, Canad. J. Math. 25 (1973), 712-726.

3. S. E. Dickson, Decomposition of modules. I. Classical rings, Math. Z. 90 (1965), 9-13. MR $32 \# 2445$.

4. P. Gabriel, Des catégories abéliennes, Bull. Soc. Math. France 90 (1962), 323-448. MR 38 \#1144.

5. O. Goldman, Rings and modules of quotients, J. Algebra 13 (1969), 10-47. MR 39 \#6914.

6. I. N. Herstein, Topics in ring theory, Univ. of Chicago Press, Chicago, Ill., 1969. MR 42 \#6018.

7. G. Krause, On fully left bounded left noetherian rings, J. Algebra 23 (1972), 8899. MR 46 \#7303.

8. J. Lambek and G. Michler, The torsion theory at a prime ideal of a right Noetherian ring, J. Algebra 25 (1973), 304-389. MR 47 \#5034.

9. L. Lesieur and R. Croisot, Algèbre noethérienne non commutative, Mémor. Sci. Math., fasc. 154, Gauthier-Villars, Paris, 1963. MR 27 \#5795.

10. G. Michler, Goldman's primary decomposition and the tertiary decomposition, J. Algebra 16 (1970), 129-137. MR 42 \#3117.

11. J. A. Riley, Axiomatic primary and tertiary decomposition theory, Trans. Amer. Math. Soc. 105 (1962), 177-201. MR 25 \#5080.

12. D. W. Sharpe and P. Vamos, Injective modules, Cambridge Univ. Press, New York, 1972.

DEPARTMENT OF MATHEMATICS, GEORGE MASON UNIVERSITY, FAIRFAX, VIRGINIA 22030 